\title{
Missing antecedents found
}

\author{
Philip Miller, Barbara Hemforth, Pascal Amsili \& Gabriel Flambard*
}

\begin{abstract}
Numerous papers have used so-called 'missing antecedent phenomena' as a criterion for distinguishing deep and surface anaphora. Specifically, only the latter are claimed to licence pronouns with missing antecedents. These papers also argue that missing antecedent phenomena provide evidence that surface anaphora involve unpronounced syntactic structure in the ellipsis site. The present paper suggests that the acceptability judgments on which the argument is based exhibit a confound because they do not take discourse conditions on VPE (a surface anaphor) and VPA (a deep anaphor) into account. Two acceptability experiments provide evidence that what is relevant to the judgments are the discourse conditions and not the presence of deep vs. surface anaphors, casting doubt on the reliability of missing antecedent phenomena as a criterion for deep vs. surface status.
\end{abstract}

Keywords. ellipsis; VP ellipsis; VP anaphora; missing antecedents; deep and surface anaphora; acceptability experiments; discourse conditions; question under discussion

1. Missing antecedent phenomena as an argument for unpronounced syntactic structure. The idea that one can use 'missing antecedent phenomena' to argue for the existence of unpronounced syntactic structure in elliptical constructions has its source in three important papers published in Linguistic Inquiry in the 1970s: Grinder and Postal 1971 (henceforth G\&P), Bresnan 1971, and Hankamer and Sag 1976 (henceforth H\&S). G\&P introduce the idea on the basis of examples like the following (adapted from their (12a,b) and (13), p.276):

(1) a. *Harry doesn't have a wife and she ${ }_{i}$ is a linguist.

b. Harry doesn't have a wife but Bill does have a wife $e_{i}$ and she $e_{i}$ is a linguist.

c. Harry doesn't have a wife but Bill does and she ${ }_{i}$ is a linguist.

d. Harry doesn't have a wife but Bill does [have a wife ${ }_{i}$ ] and she ${ }_{i}$ is a linguist.

They argue that (1-a) is ungrammatical because an "indefinite NP like a wife under scope of negation cannot serve as an antecedent for coreferent anaphors" (p.276). (1-b), on the other hand, is grammatical because the second occurrence of a wife is not under scope of negation and is thus a legitimate antecedent. The question that arises, then, is why (1-c), with Verb Phrase Ellipsis (VPE), is grammatical since it is apparently similar to (1-a) in lacking an appropriate antecedent for she. G\&P claim that this is due to the fact that (1-c) has (1-d) as its underlying structure, providing an appropriate antecedent.

Following Bresnan 1971, H\&S give an additional twist to this argument in the framework of their classical distinction between 'deep' and 'surface' anaphora. Surface anaphora are derived transformationally by deletion (in the case of ellipsis) or substitution (in the case of proforms) under identity with a syntactically present antecedent. Deep anaphora, on the other

\footnotetext{
* We would like to thank Geoff Pullum for comments on a preliminary version of this paper. This work is supported by a public grant overseen by the French National Research Agency (ANR) as part of the "Investissements d'Avenir" program (reference: ANR-10-LABX-0083). Philip Miller, Université de Paris, EA 3967 CLILLAC-ARP (philip.miller@u-paris.fr), Barbara Hemforth, Université de Paris, UMR 7710 LLF (barbara.hemforth@u-paris.fr), Pascal Amsili, Universit Sorbonne Nouvelle, UMR 8094 Lattice (Pascal.Amsili@gmx.fr), Gabriel Flambard, Université de Paris, EA 3967 CLILLAC-ARP (gabriel.flambard@gmail.com), .
} 
hand, are argued to be present as such in underlying representation and are linked to their antecedent through interpretative means. They propose that Verb Phrase Ellipsis (VPE), illustrated in (2-a) is an instance of a surface anaphor whereas Verb Phrase Anaphors (VPA) like do it, do this and do that, illustrated in (2-b), are cases of deep anaphors.

(2) a. Mary wrote a letter. She really did [VP write a letter].

b. Mary wrote a letter. She did it in just a few minutes.

In this context, they develop G\&P's argument on the basis of examples of VPE like (3) (H\&S76, (23a,b), (25), adapted from Bresnan 1971): ${ }^{1}$

(3) a. I've never ridden a camel, but Ivan's ridden a camel, and he says it stank horribly.

b. I've never ridden a camel, but Ivan has, and he says it stank horribly.

c. *I've never ridden a camel, and it stank horribly.

H\&S contrast (3-b) with examples of VPA like:

(4) *Nick the Blade didn't cut Sonny Black with a knife - Big Sam did it, and it was rusty. (Adapted from their (30)).

Their reasoning for (3) follows that of G\&P for (1): (3-b) is grammatical because the NP $a$ camel is present in deep structure, making it entirely comparable with (3-a). But (4) is ungrammatical because the VPA did it, a deep anaphor, is present as such at all levels of syntax, so that there is no occurrence of Big Sam cut Sonny Black with a knife and hence no antecedent available for $i t$.

On this basis, H\&S use the possibility of missing antecedent phenomena as a criterion for distinguishing deep and surface anaphora more generally (along with the possibility of exophoric uses and syntactically mismatched antecedents). This criterion has become a classic and has been regularly used in discussions of ellipsis up to today. ${ }^{2}$

A first important comment is that the judgments on these examples are far from clear. This was noted as early as Bresnan 1971 and is also a point made by Williams 1977 and Sag and Hankamer $1984 .^{3}$ Differential acceptability judgments are furthermore hampered by the fact H\&S do not use the same sentences to contrast VPA and VPE. Is it really clear that (5-a) is less acceptable than (3-b) and that (5-b) is more acceptable than (4)?

(5) a. I've never ridden a camel, but Ivan has done it, and he says it stank horribly.

b. Nick the blade didn't cut Sonny Black with a knife - Big Sam did, and it was rusty.

\footnotetext{
${ }^{1}$ All judgments on cited examples are those of the authors cited; as will appear below, we do not believe that a sentence like (4) is ungrammatical, but rather that it is less felicitous than the VPE alternative (given below in (5-b)) because it fails to satisfy the QUDRC discourse constraint, which we introduce below in (6). We will note this judgment using the \# sign, as opposed to the asterisk indicating ungrammaticality.

${ }^{2}$ For recent examples see, e.g., textbooks and surveys such as Lasnik and Funakoshi 2019; Merchant 2013; research papers such as Bentzen et al. 2013; Chung et al. 2011, Craenenbroeck 2010; Depiante 2019; Gergel 2009; Johnson 2001; Johnson 2016; LaCara 2016; Thompson 2014; Alcaraz 2018; Xiang et al. 2019 etc. Many of these authors point out that judgments are less clear than initially thought, but they continue to use the criterion as a diagnostic. Thompson 2014, in particular, provides a detailed defense of the criterion.

${ }^{3}$ Sag and Hankamer 1984 mention in their footnote 2, pp.342-3, that they didn't discuss the phenomenon further "because of the relative delicacy of the crucial judgments, and because of disagreement between the two authors of [the] paper over their significance".
} 
A second point is that there is something inherently surprising about the way H\&S set up the argument, as noted by Williams 1977, p.693. Specifically, they claim that personal pronouns are deep anaphors, which means, by definition, that they do not require a syntactically given antecedent. It is sufficient that the referent be accessible through conceptual structure, provided by the previous discourse or by the extralinguistic context. Since the variants with VPA in the above examples clearly do provide an antecedent in conceptual structure (e.g., in (4), if Bill "did it" then obviously there was a knife involved), it is not a priori clear why there should be any problem at all in finding a referent for it. Later work by Ward et al. 1991 and McKoon et al. 1993 has shown that the accessibility of discourse entities is affected jointly by discourse pragmatic and morphosyntactic factors. In this light, a more plausible interpretation of the argument from missing antecedents would be to frame it as saying that processing an anaphor is more difficult if the antecedent is not present in the previous discourse, in the morphosyntactic form required by the anaphoric expression, thus requiring some form of inference. Processing difficulty typically correlates with lower acceptability, ${ }^{4}$ so that rather than starring the sentences with VPA like (4), it would make more sense, in the context of the argument, to consider them grammatical but less acceptable, i.e., a ? or \# judgment (this is the position that Thompson 2014, pp.15-18, develops).

Another possible explanation for the situation might be that it is actually not the choice between VPE and VPA that determines the acceptability of the final clause with it in examples like (3-b) and (4), but some other independent factor. Specifically we suggest that a discourse condition on VPE and VPA is in fact the source of the acceptability intuitions in these cases.

2. Discourse conditions on VPE and VPA. Since the early 70s, there has been some work on the discourse conditions on VPE and VPA. However, most of that work has concentrated on VPE and furthermore has considered the way general discourse constraints interact with VPE. Typical examples of this line of research include Rooth (1992), Kehler (2002), Kertz (2013). There has been very little research on construction-specific conditions on VPE and VPA, and on the choice between the two in discourse.

In recent publications (Miller 2011; Miller and Pullum 2014; Miller and Hemforth 2014; Flambard 2018; Miller et al. 2020) we propose a construction-specific discourse condition on VPE and VPA, based on the notion of QUD (Question Under Discussion, cf., Roberts 1996; Ginzburg 2012; Onea 2016), the QUD Relevance Constraint (QUDRC): ${ }^{5}$

QUD Relevance Constraint (QUDRC)

If the QUD addressed by the anaphoric clause is conventionally introduced by the an-

\footnotetext{
${ }^{4}$ See, e.g., Fanselow and Frisch 2006 and Hofmeister et al. 2013.

${ }^{5}$ See Grant et al. 2012 for an independent suggestion that QUDs are relevant to the acceptability of VPE. In this formulation of the QUDRC, we adopt the general idea (defended by, e.g., Ginzburg 2012 and Onea 2016, see also Benz and Jasinskaja 2017) that the form of an utterance defines certain QUDs that are conventionally introduced by it, e.g., what Onea (p.9-10) calls 'Standard Potential Questions' (SPQs) and 'Primary Potential Questions' (PPQs). As noted by Onea, however, the most likely question raised by an utterance in a specific discourse context may not in fact be conventionally associated with it but rather be accommodated on the basis of extralinguistic factors. Specifically, we assume that asserting a declarative clause with propositional content $p=P(a)$ conventionally introduces two QUDs, $p \quad \vee \quad \neg p$ ? and $\lambda x . P(x)$ ?. It should also be noted that the acceptability of VPA is affected by various semantic and discourse factors independent of the QUDRC, which are beyond the scope of this paper (see, e.g., Flambard 2018, Oger 2019). This means that the QUDRC only provides a partial explanation for the acceptability of sentences with VPA.
} 
tecedent clause, VPE is acceptable and is preferred to VPA. If not, then the acceptabililty of the VPE clause correlates with the ease with which the question it addresses can be accommodated as QUD from the antecedent clause; the acceptability of VPA is inversely correlated.

It is beyond the scope of the present paper to argue in detail in favor of the QUDRC, for which we refer to the papers mentioned above. However, we illustrate the constraint and make it more intuitively plausible by briefly reviewing the results of an acceptability experiment presented in Miller et al. 2020, which can be referred to for details on the materials, methods, and results. Specifically, this experiment included conditions like the following:

VPE/ASS A-When he was in the kitchen he read the instructions.

$\mathrm{B}-\mathrm{He}$ did?

VPE/BG $\mathrm{A}-\mathrm{He}$ was in the kitchen when he read the instructions. B-He did?

Notice that A's utterance contains two clauses, one with copular be and the other with a lexical verb (read in this case). Because auxiliary do cannot be used with a copular be antecedent (be would have to be used: $A-H e$ was in the kitchen. $B-H e$ was/*did?), only the clause headed by the lexical verb is a possible antecedent. The experiment manipulated whether the antecedent was in the main clause or the temporal adjunct subordinate clause, in which case it was respectively asserted (and, as a result, conventionally introduced the QUD $p \vee \neg p$ ?) or backgrounded (and did not conventionally introduce a QUD).

We found a significant difference $(\mathrm{p}<.01)$ in acceptability for VPE depending on whether the antecedent clause is asserted or backgrounded, i.e., depending on whether the VPE utterance addresses a conventionally introduced QUD or not, which corroborates the QUDRC.

Importantly, this experiment shows that the acceptability of VPE cannot be entirely accounted for on the basis of syntactic identity. Indeed, in both the asserted and the backgrounded antecedent conditions, there is an equally distant syntactically identical antecedent available in the immediate discourse context, so that the two conditions of (7) would be expected to be judged equally acceptable. Because of this, theories invoking repair ('recycling', e.g., Arregui et al. 2006 and Frazier 2013) cannot explain the decrease in acceptability in the backgrounded condition, since there is nothing to repair (the antecedent is matched). On the other hand, the QUDRC provides a clear explanation for the acceptability data.

Having introduced the QUDRC, let us turn now to the predictions it makes on the types of sentences that will be crucial to the present paper, which are illustrated in (8):

(8) a. Sue didn't write a song. Sam did.

(VPE/Adj-)

b. Sue didn't write a song. \#Sam did it.

(VPA/Adj-)

c. Sue didn't write a song. \#Sam did for her.

$(\mathrm{VPE} / \mathrm{Adj}+)$

d. Sue didn't write a song. Sam did it for her.

(VPA/Adj+)

Notice that the antecedent clause is the same in all cases. Two factors are relevant in distinguishing the anaphoric clause, namely, whether VPE $(d o)$ or VPA (do it) is used and whether it is or is not followed by a non-contrastive adjunct (Adj+/-).

In (8), the contrasting subject in the anaphoric clause insures that the relevant convention- 
ally introduced QUD is $\lambda x . P(x)$ ?, i.e., in this case, 'Who wrote a song?' (cf. footnote 5). In (8-a) and (8-b), the anaphoric clause addresses this QUD, providing a contrastive subject. In these cases, the QUDRC predicts that VPE will be more felicitous than VPA. In (8-c) and (8-d), by contrast, the anaphoric clause goes beyond simply addressing this QUD and introduces a new QUD, namely the identity of the beneficiary of the song writing, expressed by the non contrastive PP adjunct for her. In such contexts, the QUDRC predicts that VPA is more felicitous than VPE.

It is interesting to note that the cases of missing antecedent sentences that are judged to be ill-formed in the literature typically do not respect the QUDRC. In (4), for instance, the VPA do it is used in a context where the QUDRC predicts that VPE would be preferred.

The following sections discuss two acceptability experiments that we have run. The first aims to corroborate the validity of the discourse condition given in (6), and specifically the effect of non-contrastive adjuncts; the second sheds light on whether it is the choice between VPE and VPA, or the discourse condition, or both that is relevant with respect to acceptability.

\section{Experiment 1: The discourse conditions.}

Design We created 20 experimental items of type (8), varying two binary factors: VPE vs. VPA and the presence or absence of a non contrastive adjunct (Adj+/-), i.e., four conditions. All items involve two clauses with contrasting subjects (half of the items have a typically female referring first name in the first clause and a typically male referring first name in the second clause, and vice-versa). The verb in the first clause is always a verb of creation (Levin 1993, p.172ff) with an "effected object", i.e. the referent of the object comes into existence as a result of the event. Since the clause is negated, no entity is introduced into the discourse context as a result of its utterance. In the Adj+ cases, the non contrastive adjunct in the second clause is always a PP headed by for, expressing a beneficiary adjunct, and the pronoun is either him or her, as required to refer back to the subject of the first clause. These 20 items were distributed across 4 lists in a Latin Square design (so that each subject saw only one condition of an item like (8) but an equal number of items in each condition across items), interspersed with 16 items from a separate experiment as distractors. ${ }^{6} 48$ subjects (12 per list, recruited on Amazon Mechanical Turk, https://www.mturk.com/) judged the acceptability (explained in terms of naturalness) of the second clause (underlined in the stimuli) in the context of the first on a scale of 1 to 7 . Data from one participant were eliminated from the dataset because she turned out to be bilingual. An example stimulus is included in Figure 1. Each such stimulus was followed by a yes/no question to ensure that subjects were being attentive. The experiment was run on Ibex Farm (Drummond 2014).

\footnotetext{
${ }^{6}$ These specific distractor items were chosen because they consist only in moderately to highly acceptable sentences (mean acceptability for distractors: 4.9). This is important because the materials of interest here are also all relatively highly acceptable (the average acceptability for the whole experiment being 6.14 on a scale of 1 to 7 ). A previous version of our experimental materials was run with distractors that included strongly degraded sentences, which caused the judgements on our materials to be more compressed ('end of scale compression'), making differences between conditions harder to detect statistically.
} 
progress

Emily didn't compose a melody.

Edward did.

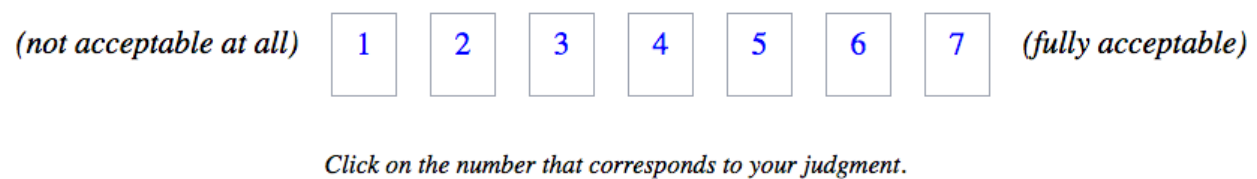

Figure 1. A typical stimulus from Experiment 1

Results The overall results are presented in Figure 2 and in Table $1 .{ }^{7}$ Sentences without adjuncts were on average judged slightly better than sentences with adjuncts (Prob $>0=.956$ ). No effect was found for the choice between VPE and VPA (Prob $>0=.68$ ). On the other hand, there is a very high probability for an interaction between Adj+/ and VPE/VPA (Prob < $0=.999)$, providing strong corroboration for the validity of the QUDRC (6): the presence of an adjunct favors VPA while its absence favors VPE. ${ }^{8}$

\begin{tabular}{l|r|r|r|r} 
Effect & Est $\beta$ & $95 \%$ ConfInt & Prob $>0$ & Prob $<0$ \\
\hline Adjunct & .29 & $-0.039-0.63$ & .956 & .044 \\
Ellipsis & .09 & $-.315-.50$ & .648 & .316 \\
Adjunct*Ellipsis & -1.59 & $-2.55--.64$ & .001 & .999
\end{tabular}

Table 1. Posterior probabilities for Experiment 1

4. Experiment 2: Missing Antecedents?. Experiment 2 was designed to shed light on whether the difference in acceptability between (3) and (4) is due to the choice between VPE and VPA,

\footnotetext{
${ }^{7}$ Acceptability ratings were submitted to a Bayesian ordinal regression model using the bmr function from the Rpackage bmrs (Bürkner 2018). Presence of Adjunct (Adj+ vs. Adj-) and Ellipsis (VPA vs. VPE) were included as centred predictors as well as the interaction between the two variables. In addition to random intercepts of items and participants, random slopes for Adjunct and Ellipsis, and their interaction were included. The model formula was "judgment $=$ adjunct $*$ ellipsis $+($ adjunct $*$ ellipsis $\mid$ subj $)+($ adjunct $*$ ellipsis $\mid$ item $)$ ". We used uninformative priors, 4 chains and 9000 iterations.

${ }^{8}$ Certain native speakers may find (8-b) (i.e., VPA/Adj-) to be less degraded in acceptability than (8-c) (VPE/Adj+), as does the native speaker author of this paper. To be more precise, both of the dispreferred conditions (8-b) and (8-c) can be improved by imagining a richer context and manipulating imagined intonation, but it seems to the author of this paper that this is easier to do in the VPA/Adj-condition. However, the naïve subjects of the acceptability experiment do not seem to clearly share this judgment (though there is a small numerical difference in the expected direction). The relatively small decrease in acceptability in the dispreferred conditions (8-b) and (8-c) can be assumed to be due to the subjects' ability to accommodate context and imagine intonation patterns that make these conditions more acceptable.
} 


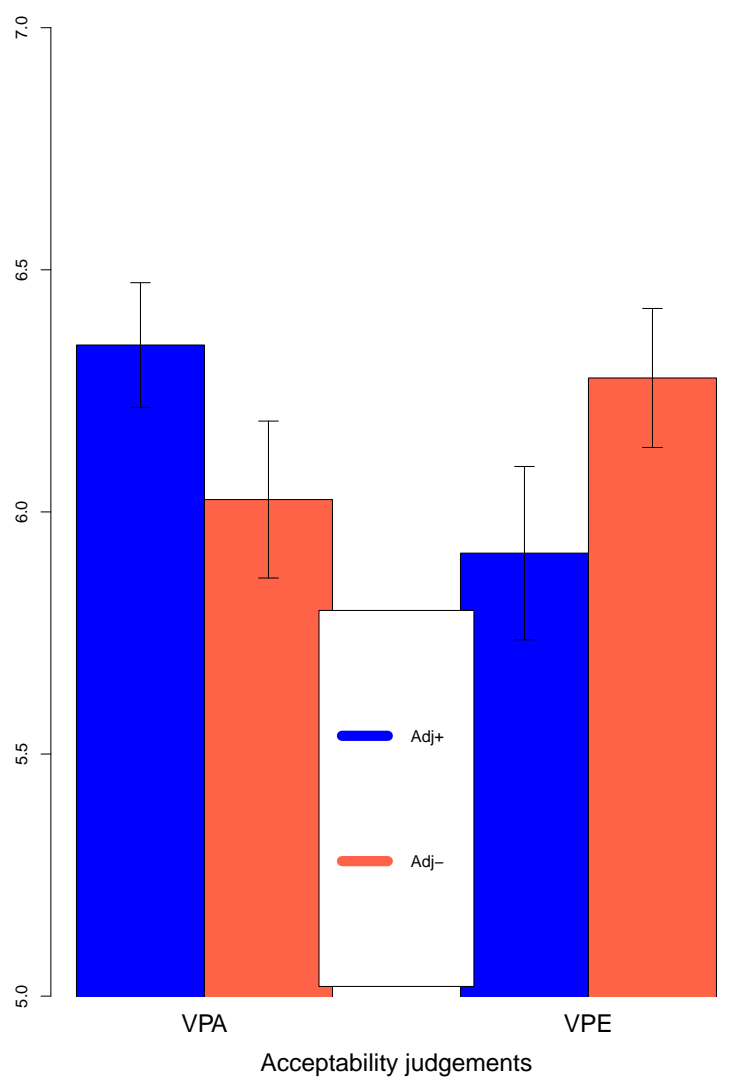

Figure 2. Experiment 1: acceptability judgments

as classically assumed, or rather to the QUDRC.

Design Once again, we used 20 items with 4 conditions, identical to those in Experiment 1, but with an additional third clause. This clause consisted in the pronoun he or she (chosen so as to be coreferent with the subject of the second clause) followed by a verb which could take the created entity as a plausible object, followed by the pronoun it (obviously interpreted as referring back to the created entity) and an adjunct, as illustrated in (9):

(9) a. Sue didn't write a song. Sam did. He sang it beautifully.

(VPE/Adj-)

b. Sue didn't write a song. Sam did it. He sang it beautifully. (VPA/Adj-)

c. Sue didn't write a song. Sam did for her. He sang it beautifully. $(\mathrm{VPE} / \mathrm{Adj}+)$

d. Sue didn't write a song. Sam did it for her. He sang it beautifully. (VPA/Adj+)

Crucial to the design is the fact that the third clause is identical across conditions. The same distractors were used as in Experiment 1 and the procedure was identical, except that 48 (different) subjects were instructed to judge the acceptability of the third clause (underlined in the stimuli) in the context of the first two. Data from one non-native speaker were eliminated from the dataset.

Expectations Because the materials are constructed so that within each item the first and third clauses do not vary, any difference in the judgment on the third clause must be due to the 
factors at play in the second clause. If, as classically assumed, the use of VPA makes it more difficult to recover an antecedent for $i t$ in the third clause, we would expect the VPA conditions (9-b) and (9-d) to be less acceptable than the VPE conditions (9-a) and (9-c). Given the results of Experiment 1, we might also expect a contamination effect from the acceptability of the second clause, so that, among the VPA conditions, (9-b) would be worse than (9-d), and among the VPE conditions, (9-c) would be worse than (9-a). These two effects are not a priori incompatible.

Results The overall results are presented in Figure 3 and in Table 2. They are mostly parallel to those of Experiment 1: Sentences without adjuncts seem to be preferred (Prob $>0=$ .95) but no preference for VPA or VPE was found (Prob $>0=.48$ ). There is a high posterior probability of an interaction between Ellipsis and presence of an Adjunct (Prob $<0=.98$ ). ${ }^{9}$ Crucially, while the experiment was sensitive to subtle differential effects of the presence of adjuncts for VPE and VPA, no main effect was found for the choice between VPE and VPA, contrary to classical expectations. Given the fact that the third sentence is identical in all conditions, the interaction we found in the judgments can only be explained as a contamination effect from the acceptability of the second clause, caused by the discourse conditions. The fact that it is not actually the third clause that is degraded also explains why the effect of the interaction is weaker.

\begin{tabular}{l|r|r|r|r} 
Effect & Est $\beta$ & $95 \%$ ConfInt & Prob $>0$ & Prob $<0$ \\
\hline Adjunct & .34 & $-0.08-0.77$ & .95 & .05 \\
Ellipsis & -.01 & $-.58-.55$ & .48 & .52 \\
Adjunct*Ellipsis & -1.11 & $-2.16--.10$ & .02 & .98
\end{tabular}

Table 2. Posterior probabilities for Experiment 2

Discussion Beyond the fact that Experiment 2 provides no evidence for a distinction between VPE and VPA in terms of their capacity to make missing antecedents available, one might argue that it actually provides some evidence against the idea of the presence of unpronounced syntactic structure in the ellipsis site. McKoon et al. (1993) show that the accessibility of discourse entities is affected not only by pragmatic but also by morphosyntactic factors: in this light (10-b) is harder to process and thus somewhat less acceptable (see fn. 4) than (10-a), because the antecedent-trigger Frenchman is not morphosyntactically identical to the antecedent required for there, namely France, making inference necessary.

(10) a. Kim is from France, but he's never lived there as an adult.

b. Kim is a Frenchman, but he's never lived there as an adult.

If VPE differs from VPA in that there is unpronounced structure in the ellipsis site, the necessary antecedent for $i t$ will be present in the appropriate morphosyntactic form in VPE (parallel to $(10-a)$ ), but not in VPA (parallel to $(10-b)$ ), where it would have to be inferred from conceptual structure. One would then expect the unpronounced structure in VPE to make the referent

\footnotetext{
${ }^{9}$ A cross-experiment analysis shows a moderate posterior probability for a three-way interaction which is not surprising since the two-way interaction seems to be numerically smaller in Experiment 2 (Adjunct*Ellipsis*Experiment: Est $\beta=-1.1406401,95 \%$ Confidence Interval $=-2.97-0.71$, Prob $>0=.11$, Prob $<0=.89$ ).
} 


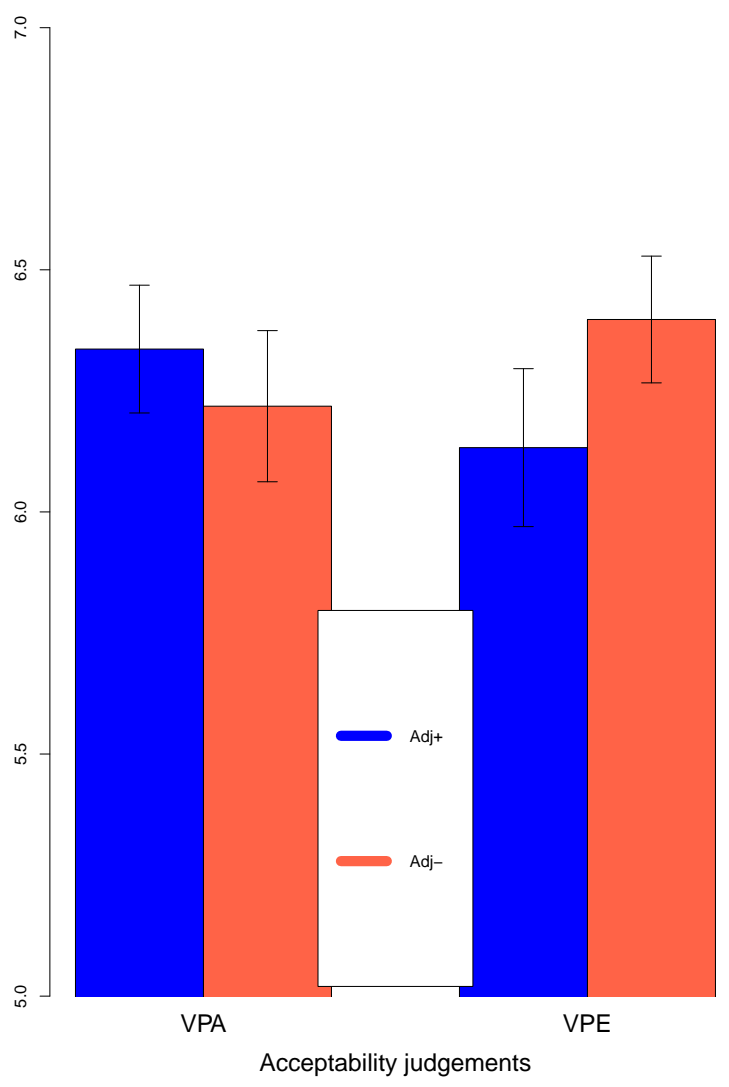

Figure 3. Experiment 2: acceptability judgments

more accessible, increasing the acceptability of the third sentence in the context of VPE, parallel to (10-a). But no such effect was found. Of course, this idea remains speculative: other factors might be at play that penalize VPE but not VPA, leading to a null effect that masks existing differences. It is thus impossible to reach a firm conclusion in the absence of more detailed knowledge about how VPA and VPE are processed.

5. Conclusion. We found a clear acceptability effect for the choice between VPE and VPA, but not in the classically expected direction. As predicted by the QUDRC, VPE is favored when the elliptical clause addresses a QUD that is conventionally established by the antecedent clause and VPA is preferred in other cases. On the other hand, no evidence was found for any effect of the choice between VPE and VPA on the acceptability of pronouns with missing antecedents. It thus appears that missing antecedent phenomena cannot serve as a reliable test for distinguishing deep and surface anaphora, as is still often the case in recent papers on ellipsis.

In this context, it is important to remember that H\&S proposed three criteria distinguishing deep and surface anaphora: (i) the acceptability of exophoric uses; (ii) the acceptability of syntactically mismatched antecedents; (iii) the possibility of missing antecedents. In the light of numerous papers ${ }^{10}$ that have shown that the first two criteria are much less reliable

\footnotetext{
${ }^{10}$ See, e.g., Miller and Pullum 2014 on exophoric uses of VPE and Ginzburg 2019 on exophoric sluicing; for mismatch in VPE, see, e.g., Kehler 2002, Kertz 2013, Miller and Hemforth 2014, Poppels and Kehler 2018, Poppels and
} 
than has been traditionally assumed, our arguments against the third criterion corroborate the general idea that the difference between deep and surface anaphora is not a simple, clearcut divide. Rather, we suggest that our present understanding of these three criteria supports the view that elliptical phenomena such as VPE and sluicing are simply anaphors (as suggested by, e.g., Hardt 1993 and many others since). Such anaphors find their antecedents in the discourse model, rather than in syntax, which does not mean that they cannot be affected by syntactic constraints or preferences. Indeed, it is well known that classical deep anaphors can be affected in this way, as illustrated in (10), which shows that the deep anaphor there prefers a locative PP as its antecedent to an NP. Similarly, the literature on the choice between deep anaphors such as it vs. this and that suggests that the syntactic form of the antecedent is relevant (see, e.g., Gundel et al. 1993, Gundel et al. 2003).

To conclude, we would like to come back on the status of the classical acceptability judgments on which the argument from missing antecedents is based. The results of our Experiment 2 suggest that Grinder \& Postal, Bresnan \& Hankamer \& Sag were correct in their acceptability judgments. This is reassuring as it shows that linguists are often able to detect small variations in acceptability with accuracy. What this squib shows is that, we are less reliable in our ability to identify what it is about a given example that explains the acceptability judgment. In this case, widening the range of data and taking discourse factors into account suggests that the difference is in fact discourse-based, rather than syntactic.

\section{References}

Alcaraz, Alejo. 2018. Deep and surface clitics in Northern Castilian Spanish. Abstract of talk presented at the GLOW conference, Budapest.

Arregui, Ana, Charles Clifton, Jr., Lyn Frazier \& Keir Moulton. 2006. Processing elided verb phrases with flawed antecedents: The recycling hypothesis. Journal of Memory and Language 55. 232-246. https://doi.org/10.1016/j.jml.2006.02.005.

Beecher, Henry. 2008. Pragmatic inference in the interpretation of sluiced prepositional phrases. San Diego Linguistics Papers 3. http://escholarship.org/uc/item/2261c0tg.

Bentzen, Kristine, Jason Merchant \& Peter Svenonius. 2013. Deep properties of surface pronouns: Pronominal predicate anaphors in Norwegian and German. The Journal of Comparative Germanic Linguistics 16. 97-125. https://doi.org/10.1007/s10828-013-9057-z.

Benz, Anton and Katja Jasinskaja. 2017. Questions under discussion: From sentence to discourse. Discourse Processes 54. 117-186. https://doi.org/10.1080/0163853X.2017.1316038.

Bresnan, Joan. 1971. A note on the notion "identity of sense anaphora". Linguistic Inquiry 2(4). 589-597.

Bürkner, Paul-Christian. 2018. Advanced Bayesian multilevel modeling with the R package brms. The R Journal 10(1). 395-411. https://doi.org/10.32614/RJ-2018-017.

Chung, Sandra, William Ladusaw \& James McCloskey. 2011. Sluicing: Between structure and inference. In Rodrigo Gutiérrez-Bravo, Line Mikkelsen \& Eric Potsdam (eds.), Representing language: Essays in honor of Judith Aissen, 31-50. Santa Cruz, CA: Linguistics Research Center, UC Santa Cruz.

Kehler 2019; for mismatch in sluicing, see, e.g., Beecher 2008, Poppels and Kehler 2020. 
Craenenbroeck, Jeroen van. 2010. The syntax of ellipsis: Evidence from Dutch dialects. Oxford: Oxford University Press.

Depiante, Marcela. 2019. Null complement anaphora. In Jeroen van Craenenbroek \& Tanja Temmerman (eds.), The Oxford handbook of ellipsis, 657-680. Oxford: Oxford University Press.

Drummond, Alex. 2014. Ibex Farm. https://spellout.net/ibexfarm/.

Fanselow, Gisbert and Stefan Frisch. 2006. Effects of processing difficulty on judgements of acceptability. In Gisbert Fanselow, Caroline Féry, Matthias Schlesewsky \& Ralf Vogel (eds.), Gradience in grammar: Generative perspectives, 291-316. Oxford: Oxford University Press.

Flambard, Gabriel. 2018. English VP anaphors: Do it, do this, do that. Paris: Université Paris Diderot dissertation.

Frazier, Lyn. 2013. A recycling approach to processing ellipsis. In Lisa Lai-Shen Cheng \& Norbert Corver (eds.), Diagnosing syntax, 485-501. Oxford: Oxford University Press.

Gergel, Remus. 2009. Modality and ellipsis: Diachronic and synchronic evidence. Berlin: De Gruyter.

Ginzburg, Jonathan. 2012. The interactive stance. Oxford: Oxford University Press.

Ginzburg, Jonathan. 2019. Exclamative sluices: Evidence for semantically-based ellipsis resolution. In Proceedings of Sinn und Bedeutung 2019. Osnabrück.

Grant, Margaret, Charles Clifton \& Lyn Frazier. 2012. The role of non-actuality implicatures in processing elided constituents. Journal of Memory and Language 66. 326-343. https://doi.org/10.1016/j.jml.2011.09.003.

Grinder, John and Paul M. Postal. 1971. Missing antecedents. Linguistic Inquiry 2. 269-312.

Gundel, Jeanette, Nancy Hedberg \& Ron Zacharski. 1993. Cognitive status and the form of referring expressions in discourse. Language 69(2). 274-307. https://doi.org/10.2307/416535.

Gundel, Jeanette, Michael Hegarty \& Kaja Borthen. 2003. Cognitive status, information structure, and pronominal reference to clausally introduced entities. Journal of Logic, Language and Information 12(3). 281-299. https://doi.org/10.1023/A:1024102420659.

Hankamer, Jorge \& Ivan A. Sag. 1976. Deep and surface anaphora. Linguistic Inquiry 7. 391426.

Hardt, Daniel. 1993. Verb phrase ellipsis: Form, meaning and processing. Philidelphia: University of Pennsylvania dissertation. Distributed as IRCS Report 93-23.

Hofmeister, Philip, T. Florian Jaeger, Inbal Arnon, Ivan A. Sag \& Neal Snider. 2013. The source ambiguity problem: Distinguishing the effects of grammar and processing on acceptability judgments. Language and Cognitive Processes 28. 48-87. https://doi.org/10.1080/01690965.2011.572401.

Johnson, Kyle. 2001. What VP ellipsis can do and what it can't, but not why. In Mark Baltin and Chris Collins (eds.), The handbook of contemporary syntactic theory, 439-479. Cambridge: Blackwell.

Johnson, Meredith. 2016. A description of verb phrase ellipsis in Hock. In Catherine Rudin and Bryan J. Gordon (eds.), Advances in the study of Siouan languages and linguistics, 287-312. Berlin: Language Science Press.

Kehler, Andrew. 2002. Coherence, reference and the theory of grammar. Stanford: CSLI Publications.

Kertz, Laura. 2013. Verb phrase ellipsis: The view from information structure. Language 89. 390 428. https://doi.org/10.1353/lan.2013.0051. 
LaCara, Nicholas J. 2016. Anaphora, inversion and focus. Amherst, MA: University of Massachusetts dissertation.

Lasnik, Howard and Kenshi Funakoshi. 2019. Ellipsis in transformational grammar. In Jeroen van Craenenbroek \& Tanja Temmerman (eds.), The Oxford handbook of ellipsis, 46-74. Oxford: Oxford University Press.

Levin, Beth. 1993. English verb classes and alternations: A preliminary investigation. Cambridge, MA: MIT Press.

McKoon, Gail, Gregory Ward \& Roger Ratcliff. 1993. Morphosyntactic and pragmatic factors affecting the accessibility of discourse entities. Journal of Memory and Language 32. 56-75. https://doi.org/10.1006/jmla.1993.1004.

Merchant, Jason. 2013. Diagnosing ellipsis. In Lisa Lai-Shen Cheng and Norbert Corver (eds.), Diagnosing syntax, 537-542. Oxford: Oxford University Press.

Miller, Philip. 2011. The choice between verbal anaphors in discourse. In Iris Hendrickx, Sobha Lalitha Devi, António Branco \& Ruslan Mitkov (eds.), Anaphora Processing and Applications: 8th Discourse Anaphora and Anaphor Resolution Colloquium, DAARC 2011 (Lecture Notes in Artificial Intelligence 7099), 82-95. Berlin: Springer.

Miller, Philip \& Barbara Hemforth. 2014. Verb phrase ellipsis with nominal antecedents. Ms. Université Paris Diderot.

Miller, Philip, Barbara Hemforth \& Geoffrey K. Pullum. 2020. Disentangling the effects of discourse conditions and mismatch on the acceptability of VP ellipsis. Paper presented at the 94th Annual Meeting of the Linguistic Society of America, New Orleans.

Miller, Philip and Geoffrey K. Pullum. 2014. Exophoric VP ellipsis. In Philip Hofmeister \& Elisabeth Norcliffe (eds.), The core and the periphery: Data-driven perspectives on syntax inspired by Ivan A. Sag, 5-32. Stanford, CA: CSLI Publications.

Oger, Kimberly. 2019. La grammaire de DO et ses emplois dans l'anaphore verbale. Paris: Sorbonne Université dissertation.

Onea, Edgar. 2016. Potential questions at the semantics-pragmatics interface. Leiden: Brill. Poppels, Till \& Andrew Kehler. 2018. Overcoming the identity crisis: Novel evidence for a referential theory of verb phrase ellipsis. Proceedings of the Annual Meeting of the Chicago Linguistic Society (CLS) 53. 403-417.

Poppels, Till and Andrew Kehler. 2019. Reconsidering asymmetries in voice-mismatched verb phrase ellipsis. Glossa 4(1). 60. https://doi.org/10.5334/gjg1.738.

Poppels, Till and Andrew Kehler. 2020. Inferential ellipsis resolution: Sluicing, nominal antecedents and the question under discussion. Paper presented at the 94th Annual Meeting of the Linguistic Society of America, New Orleans.

Roberts, Craige. 1996. Information structure in discourse: Towards an integrated formal theory of pragmatics. In Jae-Hak Yoon \& Andreas Kathol (eds.), Papers in semantics (Working Papers in Linguistics 49), 91-136. Columbus, OH: The Ohio State University.

Rooth, Mats. 1992. Ellipsis redundancy and reduction redundancy. In Steve Berman and Arild Hestvik (eds.), Proceedings of the Stuttgart Ellipsis Workshop, 1-36. Stuttgart: University of Stuttgart.

Sag, Ivan A. and Jorge Hankamer. 1984. Towards a theory of anaphoric processing. Linguistics and Philosophy 7. 325-345. https://doi.org/10.1007/BF00627709.

Thompson, Andrea. 2014. Beyond deep and surface: Explorations in the typology of anaphora. Santa Cruz, CA: University of California dissertation. 
Ward, Gregory L., Richard W. Sproat \& Gail McKoon. 1991. A pragmatic analysis of so-called anaphoric islands. Language 67. 439-474. https://doi.org/10.2307/415034.

Williams, Edwin. 1977. On deep and surface anaphora. Linguistic Inquiry 8. 692-696.

Xiang, Ming, Julian Grove \& Jason Merchant. 2019. Structural priming in production through 'silence': an investigation of verb phrase ellipsis and null complement anaphora. Glossa 4(1). 67. https://doi.org/10.5334/gjg1.726. 